\title{
Influence of anaesthetics on the production of cancer cell motogens, stromal cell-derived factor-1 and hepatocyte growth factor by fibroblasts
}

\author{
WENJING GONG ${ }^{1,2}$, TRACEY A. MARTIN ${ }^{2}$, ANDREW J. SANDERS ${ }^{2}$, \\ RACHEL HARGEST $^{2}$, AIHUA JIANG ${ }^{3}$, PING SUN ${ }^{1}$ and WEN G. JIANG ${ }^{2}$ \\ ${ }^{1}$ Department of Oncology, Yantai Yuhuangding Hospital, Medical College, Qingdao University, Yantai, \\ Shandong 264000, P.R. China; ${ }^{2}$ Cardiff China Medical Research Collaborative (CCMRC), \\ Division of Cancer and Genetics (DCG), Cardiff University School of Medicine, \\ Cardiff CF14 4XN, UK; ${ }^{3}$ Department of Anaesthesiology, Yantai Yuhuangding Hospital, \\ Medical College, Qingdao University, Yantai, Shandong 264000, P.R. China
}

Received July 20, 2020; Accepted October 12, 2020

DOI: 10.3892/ol.2020.12401

\begin{abstract}
Anaesthetics have been implicated to influence cancer cells and progression. Similarly, crosstalk between cancer cells and stromal components within the microenvironment is also an important factor driving progression. Stromal cell-derived factor-1 (SDF-1) and hepatocyte growth factor (HGF) are key chemokines/cytokines produced by fibroblasts which have been established as influential factors in cancer progression. The present study explored the capacity of anaesthetics to influence the expression of these key molecules in fibroblasts. The anaesthetics rocuronium bromide (RB), vecuronium bromide (VB), suxamethonium chloride CRS (SCC), dexmedetomidine hydrochloride (DH) and lidocaine were used to treat MRC-5 fibroblasts over a range of concentrations. Following treatment, transcript expression of SDF-1 and HGF was quantified using quantitative PCR. Treatment of MRC-5 cells with RB brought about a reduction of SDF-1 expression which was found to be significant in the $45 \mu \mathrm{g} / \mathrm{ml}$ treatment group. Treatment with the other anaesthetics brought about some alterations in SDF-1 expression but these were not found to be statistically significant. Treatment with the tested anaesthetics did not have any significant effect on HGF transcript expression within MRC-5 cells, although again some alterations were observed. The results indicated that anaesthetics may have an impact on the fibroblast component of the tumour microenvironment, potentially influencing
\end{abstract}

Correspondence to: Professor Wen G. Jiang, Cardiff China Medical Research Collaborative (CCMRC), Division of Cancer and Genetics (DCG), Cardiff University School of Medicine, Henry Wellcome Building, Heath Park, Cardiff CF14 4XN, UK

E-mail: jiangw@cardiff.ac.uk

Key words: anaesthetics, stromal cell-derived factor-1, hepatocyte growth factor, fibroblast, cancer
SDF-1 and HGF expression which in turn could influence tumour progression.

\section{Introduction}

Surgery is the most commonly used treatment for the majority of solid tumours. However, there is a growing recognition that surgery could potentially influence tumour recurrence and spread because of its effect on the immune system, the microenvironment or as a direct effect on cancer cells (1). Some retrospective studies have demonstrated that anaesthetic drugs have an impact, probably as one of multiple factors, on tumour proliferation and metastasis $(1,2)$. Some studies have shown that anaesthetics played a role in promoting cancer metastasis, whilst others demonstrate that anaesthetics could induce apoptosis and reduce cancer recurrence and metastasis $(1,3,4)$. However, a comprehensive understanding of the underlying mechanisms is still unclear.

The tumour microenvironment (TME), containing blood vessels, stroma cells and the extracellular matrix (ECM), plays a key role in cancer progression and metastasis (5). Fibroblasts, as one of the important components in the TME, play a vital role in tumour progression and metastasis (6). Fibroblasts, considered to be indolent in ECM in normal tissues become activated in response to wound healing, inflammation, or tissue damage caused by cancer cells (5). After that, stimuli including chemokines, cytokines, growth factors and exosomes released by fibroblasts can affect the TME by mediating immune cells, self-sustained activation and regulating cancer cells $(5,7)$. Stromal cell-derived factor-1 (SDF-1) and hepatocyte growth factor (HGF) are key chemokines and cytokines derived from fibroblasts. They are known to promote tumourigenesis, metastasis and drug resistance $(7,8)$. Whether anaesthetics are involved in tumour progression through the regulation of factors derived from fibroblasts remains to be clearly understood.

Rocuronium bromide (RB), vecuronium bromide (VB) and suxamethonium chloride CRS (SCC) are general intravenous 
anaesthetics (9). Studies have demonstrated that RB and SCC could enhance gastric cancer and breast cancer cell proliferation, invasion and migration and $\mathrm{VB}$ has been shown to promote the adhesiveness of gastric cancer cells $(9,10)$. Additionally, dexmedetomidine hydrochloride (DH) was demonstrated to promote cancer cell proliferation and progression (11) whereas lidocaine, widely used as a local anaesthetic, has been demonstrated to reduce cancer recurrence and help cancer sensitivity to cisplatin $(12,13)$.

In order to further explore the role of these anaesthetics in cancer progression and gain additional insights into their potential mechanisms, we explored whether different anaesthetics may play a role in regulating SDF-1 and HGF derived from fibroblasts.

\section{Materials and methods}

Cell culture. MRC-5 cells were obtained from ATCC (LGC Standards). The cells were cultured in Dulbecco's modified Eagle's medium (DMEM) (Sigma-Aldrich; Merck KGaA), supplemented with an antibiotic mixture containing penicillin, streptomycin and amphotericin B (Sigma-Aldrich; Merck KGaA) and $10 \%$ foetal calf serum (FCS) (Sigma-Aldrich), in an incubator at $37^{\circ} \mathrm{C}, 95 \%$ humidification and $5 \% \mathrm{CO}_{2}$.

Anaesthetics. The five anaesthetics RB, VB, SCC, DH and lidocaine are widely used in clinical practice. They were obtained from Sigma (Sigma-Aldrich; Merck KGaA). RB, SCC and lidocaine were diluted in phosphate-buffered saline (PBS), DH and VB were diluted in PBS containing dimethyl sulphoxide (DMSO) (Sigma-Aldrich; Merck KGaA) to generate stocks. Final concentrations were based on extrapolation from anaesthetic doses used in clinical practice and from previous cell culture experiments where available. Subsequently, RB was diluted with DMEM to $8 \mu \mathrm{g} / \mathrm{ml}, 45$ and $80 \mu \mathrm{g} / \mathrm{ml}(10)$. VB was diluted with DMEM to $1.5 \mu \mathrm{g} / \mathrm{ml}, 10$ and $15 \mu \mathrm{g} / \mathrm{ml}$ (10). SCC was diluted with DMEM to $20 \mu \mathrm{g} / \mathrm{ml}$, 100 and $200 \mu \mathrm{g} / \mathrm{ml}(10)$. DH was diluted with DMEM to $2.5 \mathrm{ng} / \mathrm{ml}, 5$ and $50 \mathrm{ng} / \mathrm{ml}(11,14-16)$. Lidocaine was diluted with DMEM to $0.1 \mathrm{mM}, 1$ and $10 \mathrm{Mm}(12,13,17-19)$. Control groups consisted of either media alone or media containing the respective final concentration of DMSO in accordance with treatment preparation.

Treatment of MRC-5 cells with anaesthetics. MRC-5 cells were seeded into 6-well plates and cultured until 70-90\% confluent. Following this, MRC-5 cells were treated with different concentrations of anaesthetics or control solutions. Following that the 6 -well plates were incubated at $37^{\circ} \mathrm{C}$ for $1 \mathrm{~h}$.

Total RNA isolation and RNA quantification. Following treatment of MRC-5 with the respective anaesthetics, RNA was extracted using TRI reagent (Sigma-Aldrich; Merck KGaA) in accordance with the manufacturer's instructions. Following extraction and resuspension, RNA concentration was measured using a nanophotometer (Implen, Geneflow).

Reverse Transcription (RT). RNA from MRC-5 cells treated with different anaesthetics was standardised to $500 \mathrm{ng}$ and used as a template for reverse transcription to generate cDNA using a GoScript oligo (dT) Reverse Transcription kit (Promega Corp.) in accordance with the manufacturer's instructions. The reaction was undertaken in a SimpliAmp Thermal Cycler (Thermo Fisher Scientific, Inc.).

Polymerase chain reaction (PCR) and agarose gel electrophoresis. PCR was performed on sample cDNA to probe for molecules of interest. The $16 \mu \mathrm{l}$ reaction consisted of cDNA, forward primer, reverse primer (Table I), GoTaq Green Mastermix (Promega Corp.) and PCR grade water and was conducted in a SimpliAmp Thermal Cycler (Thermo Fisher Scientific, Inc.). Following this, DNA fragments were separated by agarose (Melford Laboratories Ltd.) gel electrophoresis.

Reverse transcription-quantitative PCR (RT-qPCR). The Amplifilour ${ }^{\mathrm{TM}}$ Uniprimer $^{\mathrm{TM}}$ Universal system (Intergen Company) was used to quantify relative transcript copy number as previously described (20). In brief, the reaction contained a forward primer, reverse primer (Table I), present at $1 / 10$ th concentration of the forward primer and containing an additional sequence named as the $\mathrm{Z}$ sequence (5'-ACTGAA CCTGACCGTACA-3'), Uniprimer probe, 2X Precision FAST mastermix (Primerdesign), cDNA and PCR water. All samples were run alongside standards of known transcript copy numbers, allowing generation of a standard curve and determination of relative transcript expression within each sample.

The reaction was undertaken in a StepOne Plus qPCR system (Thermo Fisher Scientific, Inc.). Additionally, sample glyceraldehyde 3-phosphate dehydrogenase (GAPDH) levels were also quantified and used to normalise transcript expression.

Statistical analysis. Statistical analysis was undertaken using a one-way analysis of variance (ANOVA), with Holm-Sidak post hoc analysis used to compare individual groups to the control, and the SigmaPlot (version 11) statistical software. Every experiment was repeated at least three times. Differences were considered statistically significant when $\mathrm{P}<0.05$. Bar charts were prepared using GraphPad Prism (version 8) software.

\section{Results}

Effect of different anaesthetics on SDF-1 transcript expression in MRC-5 cells. The impact of anaesthetic treatment on MRC-5 transcript expression was examined using PCR. The results showed that the expression of SDF-1 was generally reduced with increasing doses of RB (Fig. 1A), DH (Fig. 1B) and SCC (Fig. 1C). A slight increased SDF-1 expression was observed with increasing doses of VB (Fig. 1D). Lidocaine had little effect on SDF-1 expression in MRC-5 cells (Fig. 1E).

SDF-1 expression was further explored using qPCR. Expression in the treatment groups was calculated as a percentage of the control group, which itself was taken as having a value of $100 \%$. Similar to the PCR result, the RB treatment group showed a reduced expression of SDF-1 following treatment and this was found to be significant, with post hoc analysis indicating a significant reduction in the $45 \mu \mathrm{g} / \mathrm{ml}$ group compared with $0 \mu \mathrm{g} / \mathrm{ml}$ group $(\mathrm{P}<0.05)$ (Fig. $1 \mathrm{~F})$. Lower 
Table I. PCR and quantitative PCR primer sequences used to detect SDF-1, HGF and GAPDH.

Primer name

SDF-1 F1

SDF-1 R1

SDF-1 Zr1

HGF F9

HGF Zr9

GAPDH F8

GAPDH R8

GAPDH F1

GAPDH Zr1
Primer sequence (5'-3')

The Z sequences are presented in italics. SDF-1, stromal cell-derived factor-1; HGF, hepatocyte growth factor.
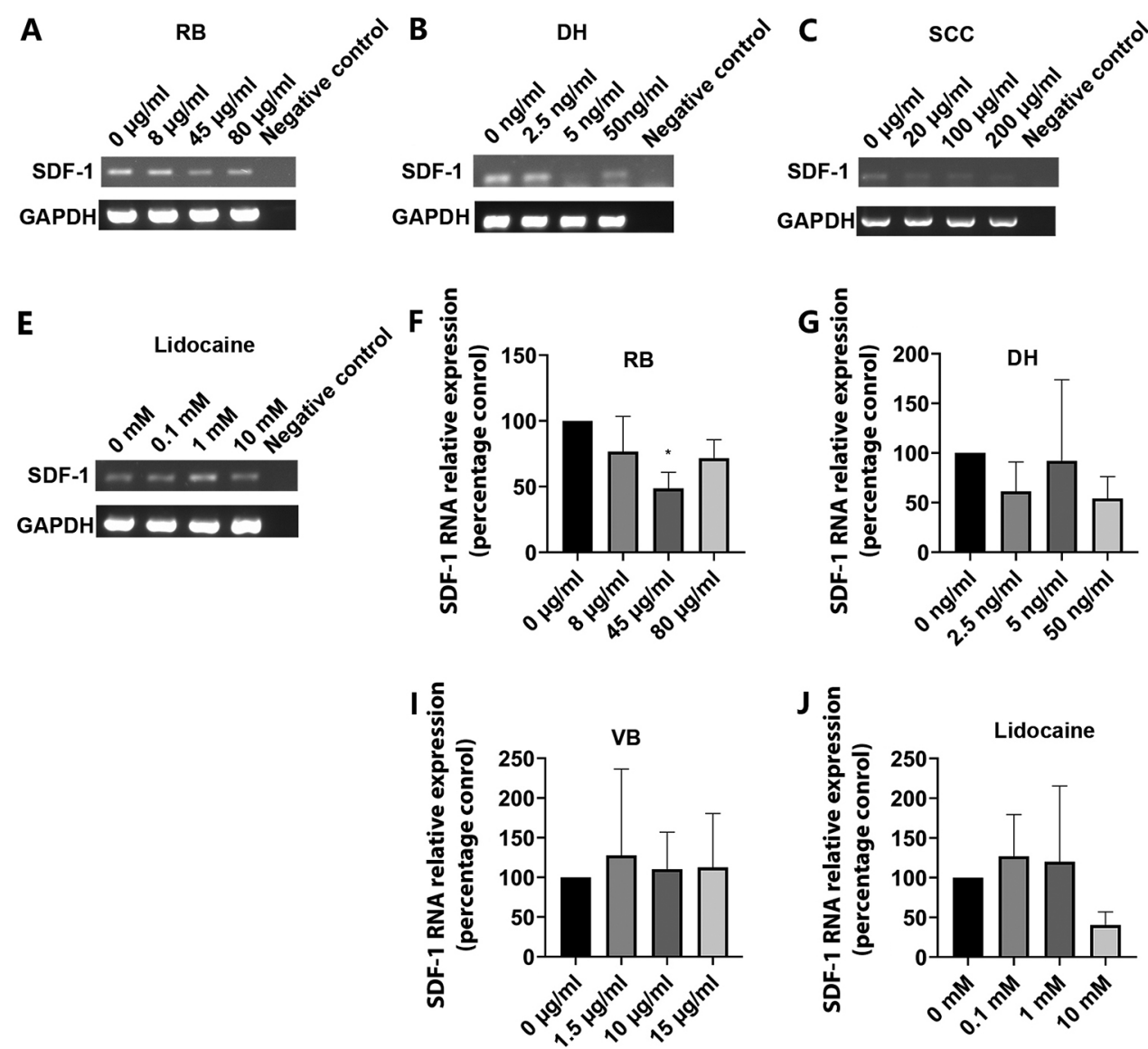
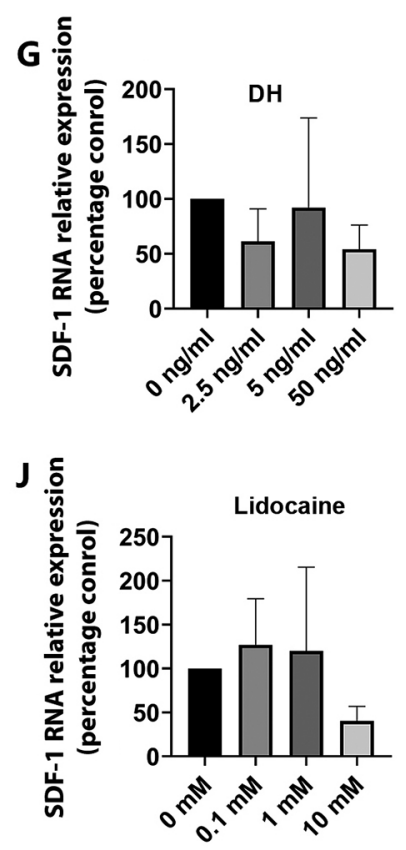
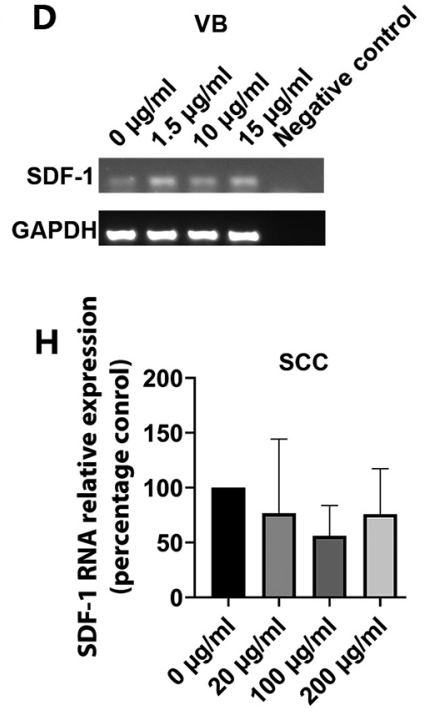

CAACGTCAAGCATCTCAAAA

TACGCTACGAAGTG

GGCTGCTTTTAACTCTGGTA

GACTGTGGTCATGAGTCCTT

Figure 1. Effect of anaesthetics on SDF-1 transcript expression in MRC-5 fibroblasts. SDF-1 transcript expression was assessed using PCR following treatment with a range of concentrations of (A) RB, (B) DH, (C) SCC, (D) VB and (E) lidocaine. Representative images are shown. Additionally, SDF-1 transcript expression was quantified using quantitative PCR following treatment with varying concentrations of (F) RB, (G) DH, (H) SCC, (I) VB and (J) lidocaine. Data are presented as the mean percentage control values $+/$ - standard error of mean. Statistical comparisons were performed using ANOVA. * $\mathrm{P}<0.05$ vs. $0 \mu \mathrm{g} / \mathrm{ml}$ control. SDF-1, stromal cell-derived factor-1; RB, rocuronium bromide; $\mathrm{DH}$, dexmedetomidine hydrochloride; SCC, suxamethonium chloride CRS; $\mathrm{VB}$, vecuronium bromide.

expression of SDF-1 was also observed following treatment with $\mathrm{DH}$, apparent at the 2.5 and $50 \mathrm{ng} / \mathrm{ml}$ concentrations, though a large degree of variability was observed in the $5 \mathrm{ng} / \mathrm{ml}$ group which limited statistical significance (Fig. 1G). Similarly, SCC generally decreased SDF-1 expression over increasing concentrations, but this was variable and did not reach statistical significance $(\mathrm{P}>0.05$; Fig. $1 \mathrm{H})$. No significant difference in SDF-1 expression was noted following treatment with VB (Fig. 1I). Lidocaine had variable effects, increasing SDF-1 expression at the 0.1 and $1 \mathrm{mM}$ concentration but brining about a large reduction at $10 \mathrm{mM}$, though ANOVA analysis indicated no significance within the group (Fig. 1J). 

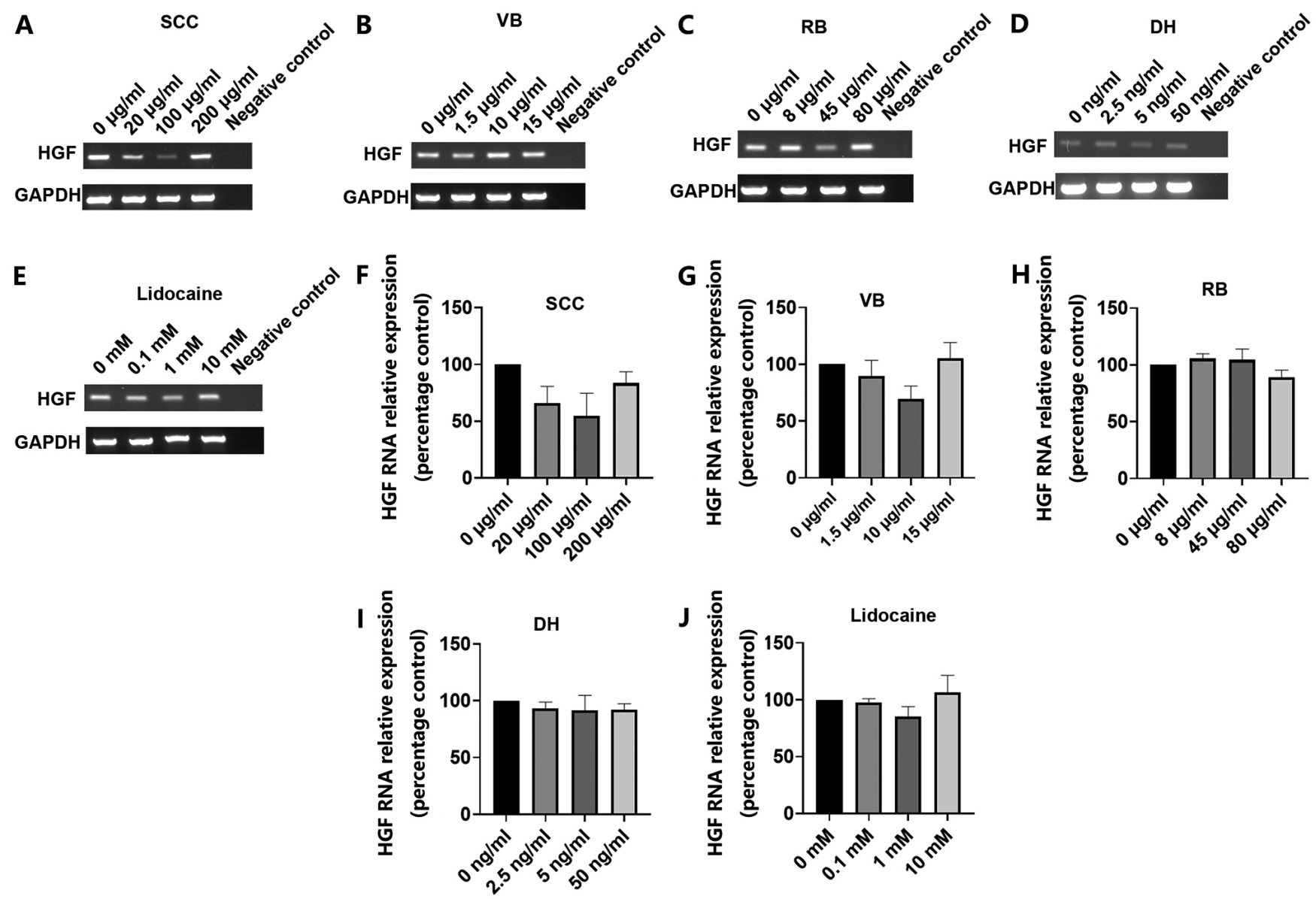

Figure 2. Effect of Anaesthetics on HGF transcript expression in MRC-5 fibroblasts. HGF transcript expression was assessed using PCR following treatment with a range of concentrations of (A) SCC, (B) VB, (C) RB, (D) DH and (E) lidocaine. Representative images are shown. HGF transcript expression was also quantified using quantitative PCR following treatment with varying concentrations of (F) SCC, (G) VB, (H) RB, (I) DH and (J) lidocaine. Data are presented as the mean percentage control values +/- standard error of mean. Statistical comparison were performed using ANOVA. HGF, hepatocyte growth factor; $\mathrm{RB}$, rocuronium bromide; DH, dexmedetomidine hydrochloride; SCC, suxamethonium chloride CRS; VB, vecuronium bromide.

Effect of different anaesthetics on HGF expression in MRC-5 cells. Conventional PCR analysis demonstrated reduced HGF transcript expression at the lower 20 and $100 \mu \mathrm{g} / \mathrm{ml}$ concentrations but no difference compared to the control at the $200 \mu \mathrm{g} / \mathrm{ml}$ treatment concentration of SCC treatment (Fig. 2A). However, $\mathrm{VB}, \mathrm{RB}, \mathrm{DH}$ and lidocaine treatment generally produced little or no change in HGF transcript levels in MRC-5 cells (Fig. 2B-E).

Similarly, qPCR analysis was also used to explore HGF transcript expression following treatment with the various anaesthetics. Expression in the treatment groups was calculated as a percentage of the control group, which itself was taken as having a value of $100 \%$. As with the PCR data, SCC treatments tended to decrease HGF expression at 20 and $100 \mu \mathrm{g} / \mathrm{ml}$ but had no effect at the higher $200 \mu \mathrm{g} / \mathrm{ml}$, although no statistical significance was observed (Fig. 2F). Interestingly, a slight enhancement of HGF expression was noted following the $15 \mu \mathrm{g} / \mathrm{ml}$ treatment of VB but ANOVA analysis demonstrated no significance within the group (Fig. 2G). No significant differences in HGF expression were identified following $\mathrm{RB}, \mathrm{DH}$ or lidocaine treatment, with little variation in HGF expression from the controls observed following treatment of MRC-5 cells with these anaesthetics (Fig. 2H-J).

\section{Discussion}

Anaesthetics are unavoidable for patients undergoing major cancer surgery. In recent years increasing evidence indicates that anaesthetics may impact patients undergoing surgery for cancer resection, highlighting the potential effect of anaesthetics on cancers (21-23). Some research has shown that anaesthetic drugs contribute to tumour proliferation and metastasis, whilst others have demonstrated the reverse response $(1,3,10,17)$. The mechanisms by which anaesthetics influence tumour growth and dissemination remain poorly understood. Our study examined five different widely used anaesthetics and how they may impact the production of cancer promoting factors from stromal cells.

In order to explore our hypothesis, we focused on five intravenous and local anaesthetics, namely RB, VB, SCC, DH and lidocaine. RB, VB and SCC are anaesthetics which act by blocking nerve impulses to muscles. Previous research has demonstrated that RB can enhance gastric cancer growth, invasion and migration and promote MDA-MB-231 breast cancer cell proliferation, migration and invasion $(9,10)$. Similarly, SCC has also been found to promote MDA-MB-231 proliferation, migration and invasion (10). VB has been noted to influence the malignant phenotype of cancer cells at a common 
concentration, whilst it has been observed to have an impact at a higher dose in gastric cancer but had very little impact on MDA-MB-231 breast cancer cells $(9,10)$. DH is a highly selective $\alpha 2$-adrenergic receptor agonist (1). $\alpha 2$-adrenergic receptors have been shown to be expressed by human breast cancer cells (24) and DH has been shown to promote tumour growth and metastasis in murine mammary tumours (25). It was also demonstrated to increase cell proliferation, migration and survival by activating $\alpha 2$-adrenoceptors in lung carcinoma (16). Lidocaine acts by blocking voltage-gated sodium channels (VGSC) on the nerve cell membrane. Several studies have demonstrated that lidocaine can inhibit growth, invasion, metastasis and promote apoptosis in lung adenocarcinoma cells, breast cancer and hepatocellular cancer cells $(19,26,27)$.

Until now, a number of mechanisms have been implicated in relation to how other anaesthetic drugs can impact on the tumour process. For instance, it has been demonstrated that propofol can inhibit the migratory capacity of cervical cancer cells by altering their morphology by regulating cytoskeletal structural stability and affecting the membrane ultrastructure (28). Additionally, it has also been indicated to induce apoptosis and inhibit growth and migration by influencing a number of different pathways. In colorectal cancer cells propofol was shown to influence the phosphatidylinositol 3-kinase (PI3K)/protein kinase B (AKT) pathway and in pancreatic cancer cells it was found to regulate miR-21/Slug signals, where Slug has been implicated as a zinc finger transcriptional repressor known to promote cancer cell invasion and survival (29). Morphine has been shown to prompt breast cancer cell migration by inducing overexpression of neuroepithelial cell transforming 1 (NET1) which is important in cell migration (30). Local anaesthetics such as lidocaine and ropivacaine have been suggested to induce non-small cell lung cancer (NSCLC) cell apoptosis, to be involved in apoptotic pathways and mitogen activated protein kinase (MAPK) pathways and to inhibit cell growth by arresting cell cycles at G0/1 phase through downregulating cyclin D1 which plays a vital role in $\mathrm{G} 1$ to $\mathrm{S}$ phase progression (17).

It appears that anaesthetics can influence cancer activity, not only via cancer cells directly but also via an indirect impact. It is known that surgical tumour resection can sometimes induce tumour recurrence and metastasis. One of the reasons is that surgery can inhibit the immune system and also affects the inflammatory system (1). Propofol has been proven to protect the immune system from being suppressed $(31,32)$. Hence, this may represent one method through which propofol could inhibit cancer growth and migration. Cell experiments and retrospective studies have suggested that DH engages in promoting tumour activity and is associated with reduced inflammatory cytokine release and regulation of the immune system (33). For example, DH could promote tumour metastasis and angiogenesis by expanding monocytic myeloid-derived suppressor cells (MDSC), and monocytic MDSC have the capacity to inhibit $\mathrm{T}$ cell proliferation and produce the proangiogenic factor vascular endothelial growth factor (VEGF) (11). Thereby, cancer progression is influenced not only by tumour cells themselves but also the contributions of components in the TME. Taken together, a full understanding of the mechanism of anaesthetics in cancer is crucial for selecting an effective treatment.
The TME is key in influencing the development and dissemination of cancer. It consists of the ECM, cytokines and stromal cells including fibroblasts, endothelial cells and immune cells (34). Fibroblasts, as the key members of the stroma, play a crucial role in tumour-stroma interaction (7). Cancer associated fibroblasts (CAF) can release a number of growth factors, cytokines, chemokines and other stimuli, most of which promote tumour progression, though some inhibit tumour function $(35,36)$. SDF-1 known as chemokine (C-X-C motif) ligand 12 (CXCL12) is a chemokine secreted by fibroblasts and engages in tumourigenesis and metastatic activity in different types of cancers, such as breast cancer (37), colorectal cancer (38) and lung cancer (39). The chemokine (C-X-C motif) receptor 4 (CXCR4), a SDF-1 receptor, has been found to be overexpressed in over 30 types of tumours (8). Its role in promoting tumour growth, migration and angiogenesis is achieved via the CXCL12/CXCR4 axis (8). HGF, released by tumour and fibroblasts, is a key cytokine that binds its receptor c-MET on different tumour cells. It modulates tumour processes such as proliferation, motility, angiogenesis, invasion and metastasis through the HGF/c-MET signalling pathway (40). Both are key factors derived from fibroblasts and contribute to tumour progression in the TME. In the current study we explored how the expression of these key factors in fibroblasts could be influenced after treatment with anaesthetic drugs.

Previous in vitro studies showed that RB promoted breast cancer and gastric cancer cell proliferation, migration and invasion $(9,10)$. SCC also increased the breast cancer cells' malignant phenotype but this was not seen in gastric cancer (10). VB had little impact on gastric and breast cancer cells activity $(9,10)$. In vitro experiments have suggested that $\mathrm{DH}$ contributed to cell proliferation and metastasis. Similar results were reported in vivo, which showed that $\mathrm{DH}$ also participated in the promotion of tumour growth $(41,42)$. Several studies proved that lidocaine could inhibit tumour cell proliferation, invasion and migration $(19,26,27)$. In our research, we explored the effect of $\mathrm{RB}, \mathrm{VB}, \mathrm{SCC}, \mathrm{DH}$ and lidocaine on the production of SDF-1 and HGF in MRC-5 cells. SDF-1 was significantly reduced by $\mathrm{RB}$ treatment at a concentration of $45 \mu \mathrm{g} / \mathrm{ml}$. The DH treatment group also showed lower SDF-1 transcript levels at concentrations of 2.5 and $50 \mathrm{ng} / \mathrm{ml}$, although there was a large degree of variability in the $5 \mathrm{ng} / \mathrm{ml} \mathrm{DH}$ treatment group. SCC treatment resulted in a similar trend to $\mathrm{RB}$ treatment, although it did not reach statistical significance. SCC and VB treatment appear to have similar trends on HGF expression. It seemed that HGF transcript expression was reduced at the lower 20 and $100 \mu \mathrm{g} / \mathrm{ml}$ concentrations of SCC treatment but little impact on expression was seen at the $200 \mu \mathrm{g} / \mathrm{ml} \mathrm{SCC}$ concentration compared to the control group. VB treatment also appeared to decrease HGF expression at 1.5 and $10 \mu \mathrm{g} / \mathrm{ml}$ concentrations but no effect at the higher $15 \mu \mathrm{g} / \mathrm{ml}$ concentration. Our study showed that lidocaine resulted in a large reduction of SDF-1 expression at $10 \mathrm{mM}$ concentration but had little impact at the other concentrations and no impact on HGF expression in MRC-5 fibroblast cells. Previous studies proved that RB, SCC and DH act in a promotive role for tumour growth, invasion and migration and lidocaine is an inhibitor of tumour progression. However, our current work demonstrates that some anaesthetics may inhibit SDF-1 and HGF expression to some extent in MRC-5 and hence 
could subsequently impact tumour proliferation, migration and angiogenesis in response to such factors. Previous studies explored the impact of the anaesthetics directly on cancer cells, whereas here our work has focused on the implications on the stromal components. It is likely that both aspects are key in the overall response of the tumour to such anaesthetics. Multiple factors should be taken into consideration when exploring the impact of anaesthetics on the tumour process. Therefore, additional complex, co-culture and in vivo assessment is needed to further understand the full significance.

There are, however, limitations to our research. Firstly, we used only one type of fibroblast cell, MRC-5, which may not be representative of all fibroblasts in vivo. Secondly, the impact of the various anaesthetics on the expression of SDF-1 and HGF were investigated at the transcript level in this initial study. Whilst this raises an important relationship of significant importance, both HGF and SDF-1 are secreted proteins. Therefore, the findings reported in this manuscript represent preliminary data. It is now of fundamental importance to further examine this relationship at the protein level and, importantly, in the context of the impact on secretion and bioactivity of such factors in the microenvironment, to validate these initial findings. This warrants further biochemical investigation to fully establish the impact of anaesthetics on fibroblast-cancer cell signalling. Thirdly, our model lacks a combination of tumour cells and TME to more fully inform the study. Additional research is required to investigate the impact of conditioned fibroblast derived medium, following anaesthetic exposure, on cancer cells. Furthermore, the current study investigated only one chemokine and one cytokine which makes it harder to represent the TME completely. Ultimately, animal studies and prospective randomized controlled trials will be required. It will be important not only to study individual drug effects on cancer growth and progression markers, but also to look at drug combinations, dosing schedules and exposure times in order to elucidate the optimum anaesthetic regimens for patients undergoing major cancer surgery in the future.

In conclusion, this study has shown that some anaesthetic drugs have the capacity to inhibit SDF-1 and HGF expression to some extent in MRC-5 fibroblasts. This alone may not result in inhibition of tumour proliferation and metastasis but does provide insights into the impact of anaesthetics on fibroblasts derived factors which may provide a new direction for improving cancer treatment. However, in vitro research cannot truly reflect the complex interaction between drugs and multiple cell types in vivo. Further studies are required to confirm the exact role of different anaesthetic agents in promoting or suppressing cancer cell proliferation and dissemination. This may open up a novel way in which cancer surgery could be optimised in future to reduce the likelihood of recurrence and metastasis.

\section{Acknowledgements}

Not applicable.

\section{Funding}

Cardiff University China Medical Scholarship, the Outstanding Young Scholarship from Yantai Yuhuangding Hospital (grant no. YDH050719).

\section{Availability of data and materials}

The datasets used and/or analysed during the current study are available from the corresponding author on reasonable request.

\section{Authors' contributions}

WGJ, PS and AJ were involved in the study concept and study design. WG, AJS and TAM participated in data acquisition, quality control of data and data analysis. WG, RH, AJS, TAM and WGJ were involved in data interpretation, preparation, editing and critical review of the manuscript. All authors read and approved the final manuscript.

\section{Ethics approval and consent to participate}

Not applicable.

\section{Patient consent for publication}

Not applicable.

\section{Competing interests}

The authors declare that they have no competing interests.

\section{References}

1. Yang W, Cai J, Zabkiewicz C, Zhang H, Ruge F and Jiang WG: The effects of anesthetics on recurrence and metastasis of cancer, and clinical implications. World J Oncol 8: 63-70, 2017.

2. Tavare AN, Perry NJ, Benzonana LL, Takata M and Ma D: Cancer recurrence after surgery: Direct and indirect effects of anesthetic agents. Int J Cancer 130: 1237-1250, 2012.

3. Le-Wendling L, Nin O and Capdevila X: Cancer recurrence and regional anesthesia: The theories, the data, and the future in outcomes. Pain Med 17: 756-775, 2016.

4. Tazawa K, Koutsogiannaki S, Chamberlain M and Yuki K: The effect of different anesthetics on tumor cytotoxicity by natural killer cells. Toxicol Lett 266: 23-31, 2017.

5. Kalluri R: The biology and function of fibroblasts in cancer. Nat Rev Cancer 16: 582-598, 2016.

6. Ohlund D, Elyada E and Tuveson D: Fibroblast heterogeneity in the cancer wound. J Exp Med 211: 1503-1523, 2014.

7. Huang TX, Guan XY and Fu L: Therapeutic targeting of the crosstalk between cancer-associated fibroblasts and cancer stem cells. Am J Cancer Res 9: 1889-1904, 2019.

8. Ahirwar DK, Nasser MW, Ouseph MM, Elbaz M, Cuitiño MC, Kladney RD, Varikuti S, Kaul K, Satoskar AR, Ramaswamy B, et al: Fibroblast-derived CXCL12 promotes breast cancer metastasis by facilitating tumor cell intravasation. Oncogene 37: 4428-4442, 2018.

9. Jiang A,Zhao H, Liu X, Yu M, Chen J and Jiang WG: Comparison of different muscle-relaxant anesthetics on growth, migration and invasion of gastric cancer cells. Anticancer Res 37: 4371-4378, 2017.

10. Jiang A, Zhao H, Cai J and Jiang WG: Possible effect of muscle-relaxant anaesthetics on invasion, adhesion and migration of breast cancer cells. Anticancer Res 36: 1259-1265, 2016.

11. Su X, Fan Y, Yang L, Huang J, Qiao F, Fang Y and Wang J: Dexmedetomidine expands monocytic myeloid-derived suppressor cells and promotes tumour metastasis after lung cancer surgery. J Transl Med 16: 347, 2018.

12. Sun $H$ and Sun Y: Lidocaine inhibits proliferation and metastasis of lung cancer cell via regulation of miR-539/EGFR axis. Artif Cells Nanomed Biotechnol 47: 2866-2874, 2019.

13. Zhang L, Hu R, Cheng Y, Wu X, Xi S, Sun Y and Jiang H: Lidocaine inhibits the proliferation of lung cancer by regulating the expression of GOLT1A. Cell Prolif 50: e12364, 2017. 
14. Deng F, Ouyang M, Wang X, Yao X, Chen Y, Tao T, Sun X, Xu L, Tang J and Zhao L: Differential role of intravenous anesthetics in colorectal cancer progression: Implications for clinical application. Oncotarget 7: 77087-77095, 2016.

15. Bao F, Kang X, Xie Q and Wu J: HIF- $\alpha / P K M 2$ and PI3K-AKT pathways involved in the protection by dexmedetomidine against isoflurane or bupivacaine-induced apoptosis in hippocampal neuronal HT22 cells. Exp Ther Med 17: 63-70, 2019.

16. Wang C, Datoo T, Zhao H, Wu L, Date A, Jiang C, Sanders RD, Wang G, Bevan C and Ma D: Midazolam and dexmedetomidine affect neuroglioma and lung carcinoma cell biology in vitro and in vivo. Anesthesiology 129: 1000-1014, 2018.

17. Wang HW, Wang LY, Jiang L, Tian SM, Zhong TD and Fang XM: Amide-linked local anesthetics induce apoptosis in human non-small cell lung cancer. J Thorac Dis 8: 2748-2757, 2016.

18. Piegeler T, Schlapfer M, Dull RO, Schwartz DE, Borgeat A, Minshall RD and Beck-Schimmer B: Clinically relevant concentrations of lidocaine and ropivacaine inhibit $\mathrm{TNF} \alpha$-induced invasion of lung adenocarcinoma cells in vitro by blocking the activation of Akt and focal adhesion kinase. Br J Anaesth 115 784-791, 2015

19. Xing W, Chen DT, Pan JH, Chen YH, Yan Y,LiQ, Xue RF, Yuan YF and Zeng WA: Lidocaine induces apoptosis and suppresses tumor growth in human hepatocellular carcinoma cells in vitro and in a xenograft model in vivo. Anesthesiology 126: 868-881, 2017.

20. Owen S, Ye L, Sanders AJ, Mason MD and Jiang WG: Expression profile of receptor activator of nuclear- $\kappa \mathrm{B}$ (RANK), RANK ligand (RANKL) and osteoprotegerin (OPG) in breast cancer. Anticancer Res 33: 199-206, 2013.

21. Hooijmans CR, Geessink FJ, Ritskes-Hoitinga M and Scheffer GJ: A systematic review of the modifying effect of anaesthetic drugs on metastasis in animal models for cancer. PLoS One 11: e0156152, 2016

22. Niwa H, Rowbotham DJ, Lambert DG and Buggy DJ: Can anesthetic techniques or drugs affect cancer recurrence in patients undergoing cancer surgery? J Anesth 27: 731-741, 2013.

23. Kim R: Anesthetic technique and cancer recurrence in oncologic surgery: Unraveling the puzzle. Cancer Metastasis Rev 36 : 159-177, 2017.

24. Vazquez SM, Mladovan AG, Perez C, Bruzzone A, Baldi A and Luthy IA: Human breast cell lines exhibit functional alpha2-adrenoceptors. Cancer Chemother Pharmacol 58: 50-61, 2006.

25. Szpunar MJ, Burke KA, Dawes RP, Brown EB and Madden KS: The antidepressant desipramine and $\alpha 2$-adrenergic receptor activation promote breast tumor progression in association with altered collagen structure. Cancer Prev Res (Phila) 6: 1262-1272, 2013.

26. Piegeler T, Votta-Velis EG, Liu G, Place AT, Schwartz DE, Beck-Schimmer B, Minshall RD and Borgeat A: Antimetastatic potential of amide-linked local anesthetics: Inhibition of lung adenocarcinoma cell migration and inflammatory Src signaling independent of sodium channel blockade. Anesthesiology 117 548-559, 2012.

27. Lirk P, Berger R, Hollmann MW and Fiegl H: Lidocaine timeand dose-dependently demethylates deoxyribonucleic acid in breast cancer cell lines in vitro. Br J Anaesth 109: 200-207, 2012.

28. Zhang F, Wang C, Cui Y, Li S, Yao Y, Ci Y, Wang J, Hou W, Wu A and Li E: Effects of propofol on several membrane characteristics of cervical cancer cell lines. Cell Physiol Biochem 40: $172-182,2016$
29. Liu Z, Zhang J, Hong G, Quan J, Zhang L and Yu M: Propofol inhibits growth and invasion of pancreatic cancer cells through regulation of the miR-21/Slug signaling pathway. Am J Transl Res 8: 4120-4133, 2016

30. Ecimovic P, Murray D, Doran P, McDonald J, Lambert DG and Buggy DJ: Direct effect of morphine on breast cancer cell function in vitro: Role of the NET1 gene. Br J Anaesth 107: 916-923, 2011.

31. Chen Y, Liang M, Zhu Y and Zhou D: The effect of propofol and sevoflurane on the perioperative immunity in patients under laparoscopic radical resection of colorectal cancer. Zhonghua Yi Xue Za Zhi 95: 3440-3444, 2015 (In Chinese).

32. Jaura AI, Flood G, Gallagher HC and Buggy DJ: Differential effects of serum from patients administered distinct anaesthetic techniques on apoptosis in breast cancer cells in vitro: A pilot study. Br J Anaesth 113 (Suppl 1): i63-i67, 2014.

33. Tsuchiya Y, Sawada S, Yoshioka I, Ohashi Y, Matsuo M, Harimaya Y, Tsukada K and Saiki I: Increased surgical stress promotes tumor metastasis. Surgery 133: 547-555, 2003.

34. Bissell MJ and Radisky D: Putting tumours in context. Nat Rev Cancer 1: 46-54, 2001

35. Chen $X$ and Song E: Turning foes to friends: Targeting cancer-associated fibroblasts. Nat Rev Drug Discov 18: 99-115, 2019.

36. LeBleu VS and Kalluri R: A peek into cancer-associated fibroblasts: Origins, functions and translational impact. Dis Model Mech 11: dmm029447, 2018.

37. Huang M, Li Y, Zhang $\mathrm{H}$ and Nan F: Breast cancer stromal fibroblasts promote the generation of $\mathrm{CD} 44^{+} \mathrm{CD} 24-$ cells through SDF-1/CXCR4 interaction. J Exp Clin Cancer Res 29: $80,2010$.

38. Todaro M, Gaggianesi M, Catalano V, Benfante A, Iovino F, Biffoni M, Apuzzo T, Sperduti I, Volpe S, Cocorullo G, et al: $\mathrm{CD} 44 \mathrm{v} 6$ is a marker of constitutive and reprogrammed cancer stem cells driving colon cancer metastasis. Cell Stem Cell 14: 342-356, 2014.

39. Li H, Chen Y, Xu N, Yu M, Tu X, Chen Z, Lin M, Xie B, Fu J and Han L: AMD3100 inhibits brain-specific metastasis in lung cancer via suppressing the SDF-1/CXCR4 axis and protecting blood-brain barrier. Am J Transl Res 9: 5259-5274, 2017.

40. Noriega-Guerra $\mathrm{H}$ and Freitas VM: Extracellular matrix influencing HGF/c-MET signaling pathway: Impact on cancer progression. Int J Mol Sci 19: 3300, 2018.

41. Bruzzone A, Piñero CP, Rojas P, Romanato M, Gass H, Lanari C and Lüthy IA: $\alpha(2)$-Adrenoceptors enhance cell proliferation and mammary tumor growth acting through both the stroma and the tumor cells. Curr Cancer Drug Targets 11: 763-774, 2011.

42. Bruzzone A, Piñero CP, Castillo LF, Sarappa MG, Rojas P, Lanari $\mathrm{C}$ and Lüthy IA: Alpha2-adrenoceptor action on cell proliferation and mammary tumour growth in mice. $\mathrm{Br}$ J Pharmacol 155: 494-504, 2008.

This work is licensed under a Creative Commons Attribution-NonCommercial-NoDerivatives 4.0 International (CC BY-NC-ND 4.0) License. 